\title{
Evidence-Based Robust Optimization of Pulsed Laser Orbital Debris Removal under Epistemic Uncertainty
}

\author{
Liqiang Hou, Massimiliano Vasile, Zhaohui Hou
}

\begin{abstract}
An evidence-based robust optimization method for pulsed laser orbital debris removal (LODR) is presented. Epistemic type uncertainties due to limited knowledge are considered. The Objective of the design optimization is set to minimize the debris lifetime while at the same time maximizing the corresponding belief value. The Dempster-Shafer theory of evidence (DST), which merges interval-based and probabilistic uncertainty modeling, is used to model and compute the uncertainty impacts. A Kriging based surrogate is used to reduce the cost due to the expensive numerical life prediction model. Effectiveness of the proposed method is illustrated by a set of benchmark problems. Based on the method, a numerical simulation of the removal of Iridium 33 with pulsed lasers is presented, and the most robust solutions with minimum lifetime under uncertainty are identified using the proposed method.
\end{abstract}

\section{Introduction}

The vast majority of the objects larger than $1 \mathrm{~cm}$ diameter in low-Earth orbit consists of space debris, remnants of larger man-made objects [16, 14]. Some of them will drop out of orbit and finally re-enter the atmosphere due to atmospheric effects, but most of them may stay in the orbit for numbers of years if no operation of debris removal is implemented. In the past decades, the amount of space debris orbiting Earth

Liqiang Hou

Xian Satellite Control Center, Xian, China 710043, e-mail: houliqiang2008@gmail.com

Massimiliano Vasile

Department of Mechanical and Aerospace Engineering, University of Strathclyde, 75 Montrose Street, G1 1XJ,Glasgow, United Kingdom, e-mail: massimiliano.vasile@ strath.ac.uk

Zhaohui Hou

School of Information and Communication Engineering, Beijing University of Posts and Telecommunications, Beijing, China 100876 
has grown exponentially. The growing space debris seriously threaten the safety of spacecrafts on orbit, especially in the near-Earth space, in which the probability of collision with debris increases steadily. To tackle the issues, researchers proposed a series of methods to reduce the threat of debris by collision avoidance maneuver and active debris removal. Recent proposals to reduce the amount of debris include electrical currents, slingshots, solar sails, electro dynamic tethers etc. Some of these proposals are still hypothetical, some of them are operational and will be implemented in the near future. Among them the Pulsed Laser Orbital Debris Removal (LODR) has attracted the attention of many researchers, whose feasibility has been demonstrated in some recent missions.

The ground-based Laser Orbital Debris Removal (LODR) system delivers a pulsed laser on the target object to deorbit the target. The pulsed laser is delivered at a pre-determined angle such that the plasma jet generated by the laser, slows down the target's velocity, and lowers its perigee altitude. A typical project using LODR includes the Orion Project, conducted by NASA and the USAF in 1995-96 (Campbell 1996). In [13], effects of irregular shapes on the laser debris removal are investigated. In [14], a method of debris collision avoidance using laser radiation is proposed. Simulation results show that promising results of range displacement for the LEO debris objects could be achieved.

In this chapter, a robust optimization method for determining the targets of pulsed Laser Orbital Debris Removal (LODR) under uncertainty is presented. Due to limited knowledge, actual values of the debris characteristics parameters, e.g. mass, dimensions, and ablation rate, may not be available before the design optimization. In most cases, the information on the uncertainties are given in the form of interval distributions. To minimize the impact of the uncertainties, an evidence-based robust design optimization method can be used. The robust design optimization takes into account the epistemic uncertainties, optimizes the LODR performance and maximizes its belief value [22]. A numerical lifetime predictor is used to evaluate lifetimes of the debris before and after the laser pulses.

Robust design optimization is complex and expensive. In design optimization of LODR, the uncertainties, such as the dimensions and masses of the debris are hard to be modelled using the conventional continuous distribution model. With DST, both the epistemic and aleatory uncertainty can be properly modeled [27, 6]. However, the number of focal elements needed to be explored in the design optimization grows exponentially with the dimension of the uncertain space, and soon becomes prohibitively expensive if a number of uncertainties are involved [21]. Another problem in the design optimization relates to lifetime prediction of the debris in the LODR. The high accuracy numerical debris lifetime prediction model is computationally expensive, and is repetitively used during the search of the optimal solutions. Therefore, a strategy that reduces the computational cost due to the numerical lifetime prediction, while maintaining the accuracy, is required.

The evidence-based robust design optimization of LODR is formulated as a multi-objective optimization problem (MOP). Unlike the conventional design optimization, with the evidence based robust optimization, a step-like Pareto front should be obtained. Therefore, some new algorithms and techniques for the de- 
sign optimization should be developed. Accordingly, the remainder of the chapter is structured as follows: Section 2 presents the general framework of design optimization of pulsed LODR. In section 3, a multi-objective optimization (MOO) algorithm with Tchebysheff decomposition and Gaussian Predictor is developed. A Kriging based surrogate is used to reduce the cost due to the numerical debris lifetime prediction. Discussions of the strategy to incorporate the evidence computation into the design optimization are presented in this section too. Section 4 presents a numerical simulation of a LODR example problem under epistemic uncertainties, and finally section 5 concludes the book chapter.

\section{Design Optimization of Laser Orbital Debris Removal (LODR)}

\subsection{Pulsed LODR}

Fig. 1 shows schematically the geometry of laser-target interaction of pulsed LODR and debris. Series of Multi-kJ laser pulses are delivered at the target debris to decelerate the debris velocity and finally re-enter it into the atmosphere. The velocity variation obtained with the pulse laser is $[13,17]$

$$
\Delta v=\frac{\eta_{0} C_{m} \Phi}{\mu}
$$

where $\eta_{0}$ is the efficiency factor for the combined effects of improper thrust direction, target shape, target tumbling, etc. $\mu$ is the target areal mass density, $\Phi$ is the laser fluence, $C_{m}$ is mechanical coupling coefficient to the laser pulse energy, and defined as $[17,20]$

$$
C_{m}=\frac{p \tau}{\Phi}=\frac{p}{I} \quad\left(\mathrm{PaW}^{-1} \mathrm{~m}^{-2} \quad \text { or } \quad \mathrm{N} / \mathrm{W}\right)
$$

where $p$ is the ablation pressure delivered to the target, $I$ is the laser intensity, $\tau$ is the duration of the pulse, and $\Phi$ is the laser fluence.

The laser pulses are delivered at the target when the LODR station finds the target, and the angle between the debris velocity and the laser beam

$$
\vartheta=\arccos \left(\frac{\left|\left(\mathbf{r}-\mathbf{r}_{l}\right) \cdot \mathbf{v}\right|}{\left|\mathbf{r}-\mathbf{r}_{l}\right| \times|\mathbf{v}|}\right)
$$

is greater than a specified value, e.g. 100 degrees, where $\mathbf{r}_{l}$ is the position of LODR station, $\mathbf{r}$ and $\mathbf{v}$ are the debris position and velocity respectively.

The $\Delta v$ obtained with Eq.1 is aligned with laser beam, and the velocity variation in radial-transversal coordinates $\Delta \mathbf{v}=\left[\Delta v_{r}, \Delta v_{\theta}, \Delta v_{n}\right]$ due to the laser pulse can be computed as

$$
\left[\begin{array}{l}
\Delta v_{r} \\
\Delta v_{\theta} \\
\Delta v_{n}
\end{array}\right]=\mathbf{M}_{o i} \frac{\left(\mathbf{r}-\mathbf{r}_{\mathbf{l}}\right)}{\left|\mathbf{r}-\mathbf{r}_{\mathbf{l}}\right|} \Delta \mathbf{v}
$$


where $\mathbf{M}_{o i}$ is the transformation matrix from the earth-centred inertial (ECI) reference frame to the radial-transversal coordinate system, and $\mathbf{r}_{l}$ is the position of the ground station in ECI frame.

Given the velocity variation in radial-transversal coordinates, variations of the orbital elements due to the velocity increment can be computed ([19], Ch.12). Repeating the process until the LODR is stopped, the debris orbital elements after the LODR can be obtained. The orbit propagator can then be used to predict the debris lifetimes after the LODR.

\subsection{Debris Lifetime Prediction}

A numerical orbit propagator model can be used to predict the debris lifetime after LODR. In the numerical model, influences of the gravitational and non-gravitational forces, including Non-spherical Earth, Third-bodies, atmospheric drag, solar radiation pressure (SRP), etc. are considered.

The acceleration due to solar radiation pressure is given by

$$
\mathbf{a}=-C_{r} \frac{A}{m} \frac{K \varphi_{s}}{c}\left(\frac{1}{R_{A U}}\right)^{2} \mathbf{r}_{s}
$$

where $C_{r}, \phi_{s}$ and $c$ are coefficient of reflectivity, solar flux at $1 \mathrm{AU}$ and the speed of light respectively. $K$ represents the percentage of the sun, as seen from the object (usually 1.0), $R_{A U}$ is the distance from the object to the sun in $\mathrm{AU}$, and $\mathbf{r}_{s}$ is the unit position vector of the sun, as seen from the debris.

The acceleration due to atmospheric drag is given by

Fig. 1 Geometry of lasertarget interaction of pulsed LODR: the laser pulses are delivered at the target to lower its perigee altitude. During the LODR, the angle $\vartheta$ is greater than a specified value.

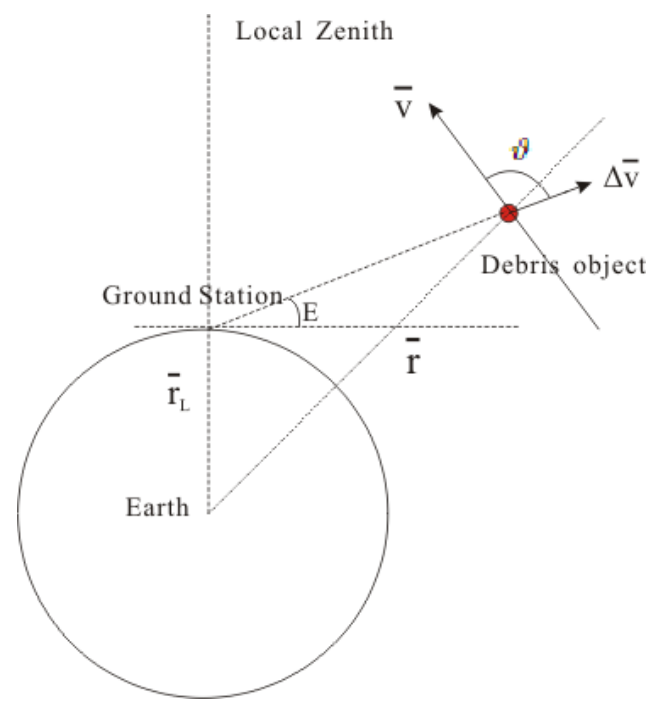




$$
\mathbf{D}=\frac{1}{2} C_{D} \frac{A}{m} \rho v^{2} \frac{\mathbf{v}}{|\mathbf{v}|}
$$

where $C_{D}, A$ and $m$ are the drag coefficient, cross-sectional area and mass, respectively. $\mathbf{v}$ is the velocity of the debris relative to the atmosphere, and $\rho$ is the atmospheric density at the location of the debris.

The drag coefficient is a dimensionless quantity which describes the interaction of the atmosphere with the surface material. The coefficient depends on several factors, including the debris shape, orientation, composition and temperature of the atmosphere, etc. A crude approximation is $C_{D}=2.2$ for compactly shaped debris [14]. A variable step-size numerical integrator is used to integrate the high fidelity dynamic equations. Error control techniques are used to manage the integration step size, increasing the computational efficiency, while preserving accuracy [19].

Although some numerical techniques, such as the variable step-size numerical integrator can be used for predicting the life-times of debris. The overall computational cost of the optimization process is expensive because one needs to compute the debris lifetimes and evaluate uncertainty impacts repetitively during the design optimization. Therefore, the cost will be huge if the numerical lifetime predictor is implemented directly in the design optimization.

\section{Evidence Based Robust Design Optimization}

\subsection{Epistemic Uncertainties and Evidence Computation}

The uncertainties of debris parameters include the cross sectional area and masses. Uncertainties of laser characteristic parameters, e.g. efficiency factor and coupling coefficient, should be taken into account too. Due to a lack of knowledge, accurate distribution of the uncertainties cannot be given in advance of the design optimization. The estimated values may be from different sources, and have different belief levels too. Therefore, in this work, an evidence-based tool is used to quantify the uncertainties. Based on the evidence theory, an evidence-based robust design optimization method is proposed.

In evidence theory, beliefs of uncertain parameters $\mathbf{u}$ are supposed to be within various intervals given by the experts. The level of confidence an expert has on an elementary proposition $E$ on the set $\mathscr{U}$ is quantified using the Basic Probability Assignment (BPA). The BPA $m(E)$ satisfies the three following axioms [6, 1]:

$$
\left\{\begin{array}{l}
m(E) \geq 0, \quad \forall E \in \mathscr{U} \\
m(\emptyset)=0, \quad \text { and } \\
\sum_{E \in \mathscr{U}} m(E)=1
\end{array}\right.
$$

Table 1 shows an example of BPA structure of uncertainties of LODR. The physical properties of debris, average mass and ablation rate can differ considerably from 
one debris to the other. In this table, estimates of uncertain intervals of the debris mass and ablation rate are given in evidence form by three experts. The confidence levels represent the perception that experts have on the specified physical properties of debris:

- Expert A is quite optimistic on his/her estimate interval of the mass. Therefore he/she assigns a high confidence of $70 \%$ to the proposition that the mass will be between $1.3 \mathrm{~kg}$ and $1.5 \mathrm{~kg}$; on the other hand, the expert is less confident about the estimate of the ablation rate and assigns a confidence level of $30 \%$ that the interval of the ablation rate is between $76 \cdot 10^{-9}$ and $80 \cdot 10^{-9}$

- While expert B, due to the data available and accuracy levels of the instruments, assigns a comparatively low confidence of $20 \%$ to the proposition that the mass will be between $1.2 \mathrm{~kg}$ and $1.7 \mathrm{~kg}$. In contrast, he/she is quite confident on his/her estimate of the laser ablation rate, and the probability assignment of the proposition that the ablation rate is between $60 \cdot 10^{-9}$ and $78 \cdot 10^{-9}$ is set to $50 \%$.

- As for Expert $\mathrm{C}$, his/her estimates of the interval of the mass and ablation rate are $[1.4,1.8]$ and $\left[65 \cdot 10^{-9}, 85 \cdot 10^{-9}\right]$ respectively, with the confidence levels of the mass $10 \%$ and ablation rate $20 \%$.

Written in mathematical terms, the statements of the estimated intervals given by the three experts can be expressed as Table 1 shows.

Table 1 Example of BPA structure of the interval based evidence sets. Estimates of the intervals of the debris mass and ablation rate are given by three experts. Corresponding confidence levels, or BPAs of the intervals are listed in the table. The BPA values show the experts' confidence in the proposition of the uncertain intervals.

\begin{tabular}{llll}
\hline Parameter & Expert & $\begin{array}{l}\text { Estimated uncertain intervals } B P A(U) \\
\left(\left[U_{l}, U_{u}\right]\right)\end{array}$ \\
\hline \multirow{3}{*}{$\operatorname{mass}(\mathrm{Kg})$} & $\mathrm{A}$ & {$[1.3,1.5]$} & 0.7 \\
& $\mathrm{~B}$ & {$[1.2,1.7]$} & 0.2 \\
& $\mathrm{C}$ & {$[1.4,1.8]$} & 0.1 \\
Ablation rate $(\mathrm{Kg} / \mathrm{J})$ & $\mathrm{A}$ & {$\left[76 \cdot 10^{-9}, 80 \cdot 10^{-9}\right]$} & 0.3 \\
& $\mathrm{~B}$ & {$\left[60 \cdot 10^{-9}, 78 \cdot 10^{-9}\right]$} & 0.5 \\
& $\mathrm{C}$ & {$\left[65 \cdot 10^{-9}, 85 \cdot 10^{-9}\right]$} & 0.2 \\
\hline
\end{tabular}

An element of $\mathbf{U} \in \mathbb{R}^{n}$ that has a non-zero BPA is named a focal element (FE). When more than one parameter is considered uncertain (e.g. $u_{1}$ and $u_{2}$ ), the BPA of the uncertain space defined by the Cartesian product of single uncertain intervals is the product of the BPA of each interval

$$
m\left(u_{1}, u_{2}\right) \in\left[a_{1}, b_{1}\right] \times\left[a_{2}, b_{2}\right]=m\left(u_{1} \in\left[a_{1}, b_{1}\right]\right) m\left(u_{2} \in\left[a_{2}, b_{2}\right]\right)
$$

Two measures quantifying the epistemic uncertain impacts, the belief and plausibility of proposition $A$, over the frame of discernment $\mathbf{U}$ can then be defined as 


$$
\begin{aligned}
& \operatorname{bel}(A)=\sum_{F E \subset A, F E \in \mathbf{U}} m(F E) \\
& p l(A)=\sum_{F E \cap A \neq \emptyset, F E \in \mathbf{U}} m(F E)
\end{aligned}
$$

where $m(F E)$ is the BPA value of the focal element $F E$, and

$$
\operatorname{pl}(A)=1-\operatorname{bel}(\bar{A})
$$

Plausibility and Belief can be viewed as the upper and lower probabilities of possible values.

Figure 2 shows a BPA structure of the interval type data given by two experts. Focal elements of the BPA structure, generated by the Cartesian product of single uncertain intervals, are computed and listed in the lower part of the table. The intersection of two intervals is defined by the maximum of the two lower bounds and the minimum of the two upper bounds. With calculation of Dempsters rule, using eq.8 eq.11, cumulative belief and plausibility value of the proposition that the data is less than a specified value can be obtained. Figure 3 shows the belief and plausibility values. As the figure shows, the belief represents confidence that the proposition is true, while the plausibility represents the confidence that the proposition is possible.

Fig. 2 BPAs of the interval type data given by two experts. Corresponding focal elements are generated using eq.8. Intersection of two intervals is defined by the maximum of the two lower bounds and the minimum of

\begin{tabular}{|c|c|c|c|c|c|}
\hline \multirow{2}{*}{\multicolumn{2}{|c|}{ B }} & Interval & $\mathrm{m}$ & Interval & $\mathrm{m}$ \\
\hline & & {$[1,4]$} & 0.5 & {$[3,5]$} & 0.5 \\
\hline Interval & $\mathrm{m}$ & & & & \\
\hline$[1,4]$ & 0.333333 & {$[1,4]$} & 0.16667 & {$[3,4]$} & 0.16667 \\
\hline$[2,5]$ & 0.333333 & {$[2,4]$} & 0.16667 & {$[3,5]$} & 0.16667 \\
\hline$[3,6]$ & 0.333333 & {$[3,4]$} & 0.16667 & {$[3,5]$} & 0.16667 \\
\hline
\end{tabular}
the two upper bounds corresponding to the intersection.

Table 2 shows an example of the BPA structure associated to the uncertain parameters for a Gaussian distribution. The evidence data set consists of statistics data values of the debris and ablation rate, and three experts express their own opinion of the estimated intervals of the mean value and standard deviation. BPA values are assigned to each data set to show the expert's confidence level of the estimated data set. In the table, if $\mu_{1}^{i}=\ldots=\mu_{m}^{i}$, the parameter $\mu^{i}$ can be removed because the parameter is a deterministic parameter. The table now consists of intervals of $\sigma$ and associated BPA values. Therefore, the table can be seen as a variation of Table 1 . Belief and plausibility of the uncertain intervals can be computed in a way similar to Table 1. 
Fig. 3 Calculation of Dempsters rule using expression eq. 8 - eq.11. Cumulative plausibility and belief values of the proposition that the data is less than a specified value are computed by adding up the masses associated to the focal elements that partially or totally support the proposition. BPA structure of the data is from Figure 2.

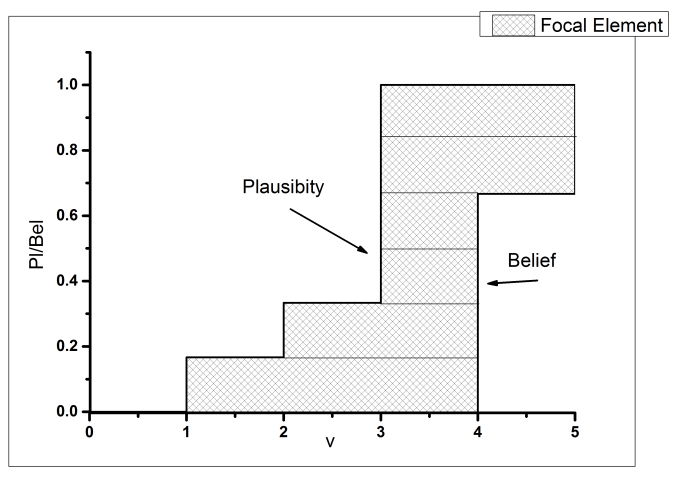

Table 2 Another example of BPA structure. This time, the BPA structure consists of estimated values, deviations and corresponding BPAs. Three experts express their opinion of estimated interval of the mean value and standard deviation of the uncertain mass and ablation rate.

\begin{tabular}{llllll}
\hline Parameter & Description & $\mu$ & $\sigma_{l}$ & $\sigma_{u}$ & BPA \\
\hline \multirow{3}{*}{$u(\mathrm{~kg} / \mathrm{J})$} & \multirow{3}{*}{ Ablation rate } & $80 \cdot 10^{-9}$ & $10 \cdot 10^{-10}$ & $20 \cdot 10^{-10}$ & 0.40 \\
& & $78 \cdot 10^{-9}$ & $15 \cdot 10^{-10}$ & $22 \cdot 10^{-10}$ & 0.30 \\
& $72 \cdot 10^{-9}$ & $12 \cdot 10^{-10}$ & $17 \cdot 10^{-10}$ & 0.30 \\
$m_{0}(\mathrm{~kg})$ & 1.30 & 0.05 & 0.10 & 0.30 \\
& \multirow{2}{*}{ Average mass $[12]$} & 1.50 & 0.05 & 0.12 & 0.20 \\
& & 1.42 & 0.05 & 0.08 & 0.50 \\
\hline
\end{tabular}

\subsection{Formulation of Evidence Based Robust Design Optimization}

Consider an optimization problem under uncertainty

$$
\min _{\mathbf{d} \in \mathbf{D}, \mathbf{u} \in \mathbf{U}} f(\mathbf{d}, \mathbf{u})
$$

where $f(\mathbf{d}, \mathbf{u})$ is the objective function, $\mathbf{d} \in \mathbf{D} \subset \mathbb{R}^{n}$ are the design variables and $\mathbf{u} \in \mathbf{U}$ are the interval-based set uncertainties with BPA values. Distribution of the uncertainties is given in the form of BPA structure as Table 1 shows.

To obtain the optimal robust design solutions, one needs to optimize the cost function and maximize its belief under uncertainties at the same time. Therefore, the problem can be formulated as a multi-objective optimization problem (MOP) $[6,27]$

$$
\left\{\begin{array}{c}
\max _{v \in \mathbb{R}, \mathbf{d} \in \mathbf{D}, \mathbf{u} \in \mathbf{U}} \operatorname{Bel}(f(\mathbf{d}, \mathbf{u})<\mathbf{v}) \\
\min _{v \in \mathbb{R}} v
\end{array}\right.
$$

where $v$ is the threshold to be minimized, and $B e l$ is the belief value.

Note that the evidence metric for the uncertainty impacts are related to the uncertain space only. Therefore, one can decompose the uncertain box first, recombine the 
focal elements and compute BPA values of the new focal elements in advance of the optimization. A sampling based approach can be used for this purpose. First, a set of intervals are generated using the sampling approach, new focal elements are then generated and combined using the evidence rules. The number of focal elements is set to be equal to the population size of the design variables, and each focal element is associated to one individual. Following eq.9 and eq.11, belief and plausibility of $f(\mathbf{d}, \mathbf{u})<\mathbf{v}$ can be computed as $[11,10]$

$$
\operatorname{Bel}(f(\mathbf{d}, \mathbf{u})<v)=\sum_{F E \in \mathbf{U} \mid \forall \mathbf{u} \in F E, f(\mathbf{d}, \mathbf{u})<v} m(F E)
$$

and

$$
P l(f(\mathbf{d}, \mathbf{u})<v)=\sum_{F E \in \mathbf{U} \mid \exists \mathbf{u} \in F E, f(\mathbf{d}, \mathbf{u})<v} m(F E)
$$

where $F E$ is the new focal element in the uncertain space. An evidence-based analysis tool is used to sample and recombine the focal elements [3, 18].

\subsection{MOO with Tchebysheff Decomposition and Proportional Orthogonal Decomposition}

Consider a MOP as follows

$$
\min _{\mathbf{x} \in \mathbf{D}} F(\mathbf{x})=\left[f_{1}(\mathbf{x}), \ldots f_{m}(\mathbf{x})\right]^{T} \quad x \in \mathbf{D} \subset \mathbb{R}^{n}
$$

where $\mathbf{x}$ is the design variable and $f_{i}(x) \in \mathbb{R}$ with $i=\in\{1,2, \ldots, m\}$ are the objective functions.

For search the optimal Pareto solutions of eq.16, the genetic MOO algorithms, e.g. NSGA-II [8], MOPSO, and MOEA/D [26] etc., can be used. However, the computational cost can be expensive as it may take hundreds of iterations of the MOOs to search for the optimal solutions. To resolve this issue, in this work, a new high efficiency MOO with decomposition is developed. The new MOO works as follows.

With the Tchebysheff decomposition, the MOP of eq. 16 can be formulated into a set of scalar single-objective optimization problems [7,2]

$$
\min _{\mathbf{x} \in \mathbf{D}} g(f(\mathbf{x}), \mathbf{w}, \mathbf{z})=\min _{\mathbf{x} \in \mathbf{D}} \max _{i=1, \ldots, m} w_{i}\left(f_{i}(\mathbf{x})-z_{i}\right)
$$

where $g \in \mathbb{R}$ is the Tchebysheff metric, $\mathbf{w} \in \mathbb{R}^{m}$ is the weight vector, and $z \in \mathbb{R}^{m}$ denotes the reference point

$$
z_{i}=\min _{\mathbf{x} \in \mathbf{D}} f_{i}(\mathbf{x}) \quad(i=1, \ldots, m)
$$

Initialize the population $\mathbf{x}_{j} \in \mathbb{R}^{n}, j=1, \ldots, n_{p o p}$ randomly, where $n_{p o p}$ is the population size. For each $\mathbf{x}_{j}^{(i)} \in \mathbf{X}^{(i)}$, where $\mathbf{X}^{(i)}$ is the population at $i$-th iteration, com- 
pute the Tchebysheff metrics $g_{j}^{(i)}, j=1, \ldots, n_{\text {pop }}$ using Eq.18. Select the individuals using the Tchebysheff metric, a set of non-dominated individuals can be obtained. Note that during the selection, some individuals may have the optimal Tchebysheff metrics w.r.t more than one weight vectors.

Suppose population $\mathbf{X}^{(i)}=\left\{\mathbf{x}_{j}^{(i)} \in^{n} \mid j=1, \ldots, n_{\text {pop }}\right\}$ can be described using a set of distribution parameters $\sigma^{(i)}=\left\{\sigma_{j}^{(i)}(\mathbf{x}) \in \mid j=1, \ldots, d \leq n_{\text {pop }}\right\}$, new distribution parameters $\sigma^{(i)}$ of the potential solutions based on previous $\sigma^{(i-1)}$ and Tchebysheff metric values $\mathbf{g}^{(i-1)}=\left\{g_{j}^{(i-1)} \in \mathbb{R} \mid j=1, \ldots, n_{\text {pop }}\right\}$ can be predicted as

$$
\sigma^{(i)}=\sigma^{(i-1)}+\mathbf{K}(\mathbf{g}, \sigma)\left(\mathbf{g}^{(i)}-\mathbf{g}^{(i-1)}\right)
$$

where $\mathbf{K}(\mathbf{g}, \sigma)$ is the feedback matrix, and new individuals $\mathbf{x}_{j}^{(i)} \in \mathbf{X}^{(i)}$ are generated using $\sigma^{(i)}$.

The key now is to estimate adaptively the distribution parameter $\sigma$ and feedback matrix $\mathbf{K}(\mathbf{g}, \sigma)$ with the previous $\sigma^{(i-1)}$ and metric values $\mathbf{g}^{(\mathbf{i}-\mathbf{1})}$. To tackle the problem, a Proportional Orthogonal Decomposition (POD, also called Principle Component Analysis (PCA) [25, 23]) and Gaussian estimator based technique is proposed.

Suppose in the $i$-th generation, the individuals are $\mathbf{x}_{j}^{(i)}$, a confidence ellipsoid that contains all the possible candidate individuals can be constructed. The distribution parameters $\sigma$ can be computed using Proportional Orthogonal Decomposition (POD, also called Principle Component Analysis). Given data set $\mathbf{X}=\left\{\mathbf{x}_{j} \in \mathbb{R}^{n} \mid j=\right.$ $\left.1, \ldots, n_{p o p}\right\}$ of the individuals, kernel matrix of $\mathbf{X}$ can be computed as

$$
\mathbf{C}=\sum_{j=1}^{n_{\text {pop }}}\left(\mathbf{x}_{j}-\mathbf{x}_{c}\right)\left(\mathbf{x}_{j}-\mathbf{x}_{c}\right)^{T}
$$

where $\mathbf{x}_{c}$ is the barycenter of $\mathbf{x}_{1}, \mathbf{x}_{2}, \ldots \mathbf{x}_{\text {npop }}$. The $j$-th principal component in the direction of the eigenvector associated with the $j$-th largest eigenvalue is

$$
\xi_{j}=\sqrt{\frac{\lambda_{j}}{\sum_{j=1}^{M} \lambda_{j}}} \mathbf{v}_{j}
$$

where $\mathbf{v}_{j} \in^{n}$ and $\lambda_{j} \in \mathbb{R}$ are eigenvectors and eigenvalues of $\mathbf{C}$ respectively. Candidate individuals in the ellipsoid can then be generated at random uniformly as the weighed sum of the principle components

$$
\mathbf{x}_{n e w}=\sum_{j=1}^{d}\left[\sigma_{j}(\operatorname{rand}(1,1)-0.5) \xi_{j}+\mathbf{x}_{r e f_{j}}\right]
$$

where $\sigma_{j} \in \mathbb{R}$ is the magnitude of the ellipsoids semi-axis in the direction of $\xi_{i} \in \mathbb{R}^{n}$, and $\mathbf{x}_{r e f_{j}}$ is the vector of the population centroid projected on $\xi_{i}$ 


$$
\mathbf{x}_{r e f_{j}}=\frac{\mathbf{x}_{c} \cdot \xi_{j}}{\left|\xi_{j}\right|^{2}} \xi_{j}
$$

Changing $\sigma=\left\{\sigma_{j} \mid j=1, \ldots, d \leq n\right\}$ and $\mathbf{x}_{r e f}$, the size and location of the ellipsoid can be varied.

In eq.22, the number of principle components $d$ that generates new design variables can be less than the dimension of design space, $n$, as those principle components that make little contributions to the function evaluations can be neglected and removed $[23,24,25]$.

Figure 4 schematically shows the proposed techniques using principle analysis. The population of the design variables in the design space and corresponding front in the criteria space are shown in the upper panel of the figure. Principle components of the population are listed in the figure too. Given the data set of distribution parameters $\sigma$, new individuals can be generated using eq.20 - eq.23. To obtain the optimal parameters $\sigma$, correlation between the data set $\sigma$ and Tchebysheff metrics g needs to be modeled. The lower panel of the figure shows the correlation between the data set of $\mathbf{g}$ and $\sigma$ established a priori, using a Gaussian type model.

Now the problem becomes that of determining the parameters $\sigma^{(i)}$ of the new ellipsoid of the solutions given $\mathbf{g}^{(i)}$, formerly $\sigma^{(i-1)}$ and $\mathbf{g}^{(i-1)}$. Suppose $\sigma^{(i-1)}$ and $\mathbf{g}^{(i-1)}$ are jointly Gaussian, i.e.

$$
\left[\begin{array}{l}
\sigma \\
\mathbf{g}
\end{array}\right] \sim \mathscr{N}\left(\left[\begin{array}{l}
\mu_{\sigma} \\
\mu_{\mathbf{g}}
\end{array}\right],\left[\begin{array}{cc}
\Sigma_{\sigma} & \Sigma_{\sigma \mathbf{g}} \\
\Sigma_{\sigma \mathbf{g}} & \Sigma_{\mathbf{g}}
\end{array}\right]\right)
$$

where $\mu_{\sigma}$ and $\mu_{\mathbf{g}}$ are the mean value of parameters $\sigma$ and $\mathbf{g}, \Sigma_{\mathbf{g}}, \Sigma_{\sigma \mathbf{g}}$ are the covariance of $\sigma$ and $\mathbf{g}$ respectively, then the conditional distribution of $\sigma$ given $\mathbf{g}=\left[g_{i}\right]$ is normal and has [4]

$$
\mu_{\sigma \mid \mathbf{g}}=\mu_{\sigma}+\Sigma_{\sigma \mathbf{g}} \Sigma_{\mathbf{g}}^{-1}\left(\mathbf{g}-\mu_{\mathbf{g}}\right)
$$

with the variance

$$
\Sigma_{\sigma \mid \mathbf{g}}=\Sigma_{\sigma}-\Sigma_{\sigma \mathbf{g}} \Sigma_{\mathbf{g}}^{-1} \Sigma_{\sigma \mathbf{g}}^{T}
$$

The estimator makes sense even when $\mathbf{g}$ and $\sigma$ are not jointly Gaussian, and $\Sigma_{\sigma}-\Sigma_{\sigma \mathbf{g}} \Sigma_{\mathbf{g}}^{-1} \Sigma_{\sigma \mathbf{g}}^{T} \leq \Sigma_{\sigma}$, i.e., the covariance of the estimation error is always less than the previous covariance of [15].

The new data set of $\sigma=\left\{\sigma_{j} \mid j=1, \ldots, d\right\}$ can then be generated as

$$
\sigma=\mathscr{N}\left(\mu_{\sigma \mid \mathbf{g}}, \Sigma_{\sigma \mid \mathbf{g}}\right)
$$

Putting the data set $\sigma$ into Eq.22, new individuals can be generated. Implementing the steps Eq.20-Eq.27 till the termination conditions (e.g. the number of iterations, MSE of $\mathbf{g}$ ) are met, the optimal solutions can be obtained.

Fig.5 shows simulation results of the new algorithm, POD-MOO and the conventional evolutionary algorithm, NSGA-II. The test function is bi-objective ZDT1 with 30 design variables. Population sizes of POD-MOO and NSGA-II are both set to 100 , and in the POD-MOO, only 5 principle components are used to gen- 


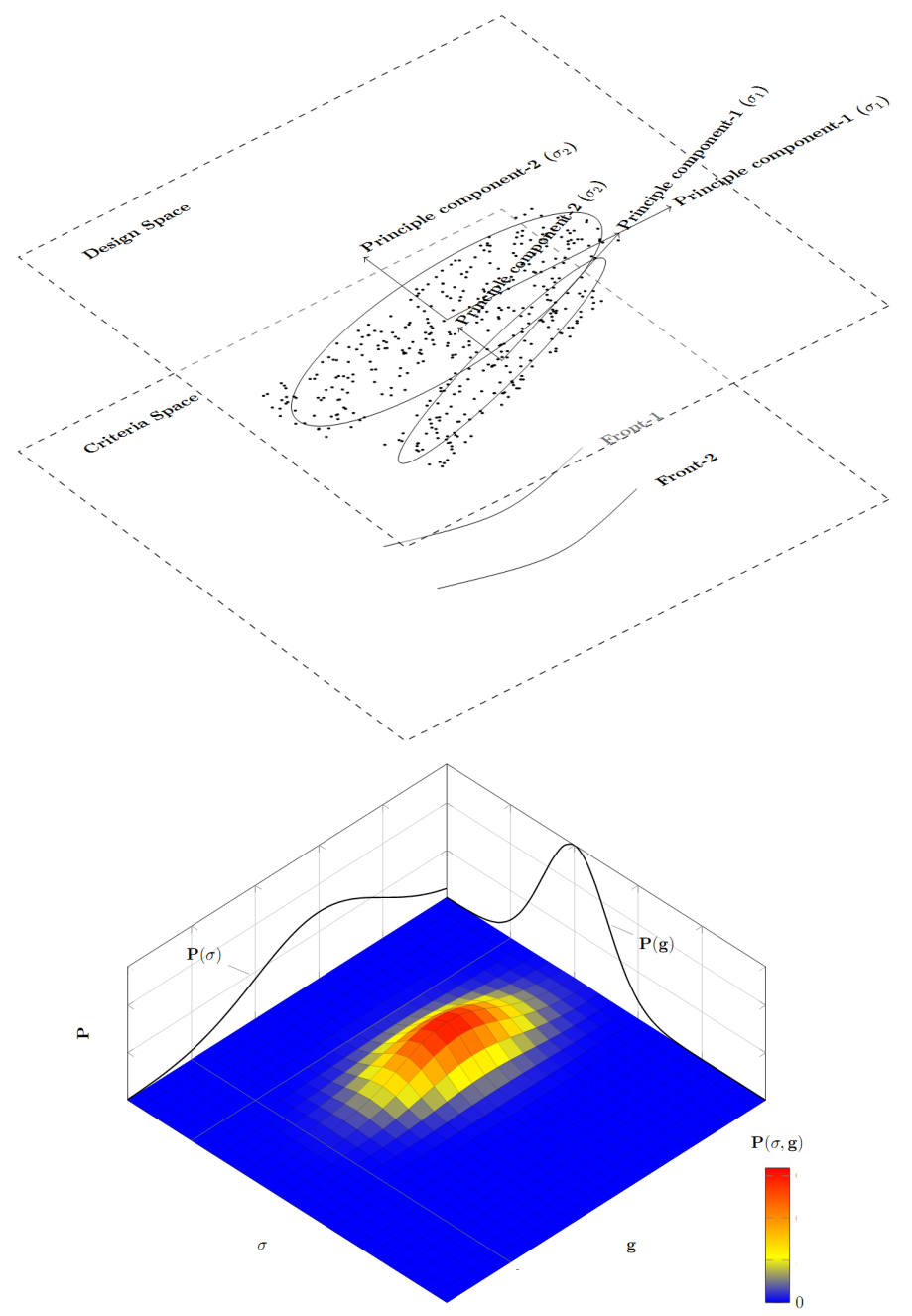

Fig. 4 Principle analysis and Tchebysheff metrics of the bi-objective optimization problem. In the upper panel, principle components of the population of design variables and corresponding fronts in the criteria space are presented. Fronts are pushed forwards by varying the control parameters $\sigma$ of principle components (eq.20 - eq.23). The lower part of the figure correlates the control parameters $\sigma$ to the data set of the metric values $\mathbf{g}$ using a Gaussian type model. Control parameters $\sigma$ to generate new individuals can be determined using the previous data set of $\sigma$ and the metric values of $\mathbf{g}$.

erate new individuals. Crossover probability and mutation probability of NSGA-II are set to $p_{c}=0.9$ and $p_{m}=1 / n$ respectively, where $n$ is the number of decision variables. The distribution indices of SBX and polynomial mutation in NSGA-II are both set equal to 20. It appears that after 20 iterations, the solutions obtained using 
POD-MOO are quite close to the true Pareto set. As for the popular MOO algorithm, NSGA-II, it may take up to hundreds of iterations before the optimal Pareto solutions are obtained.

Fig. 5 POD-MOO Vs. NSGA-II and MOEA/D. Test function: ZDT1. Iterations: 20. Population size: 200. Number of design variables:30. Function Evaluations of both algorithms: around 4000 .

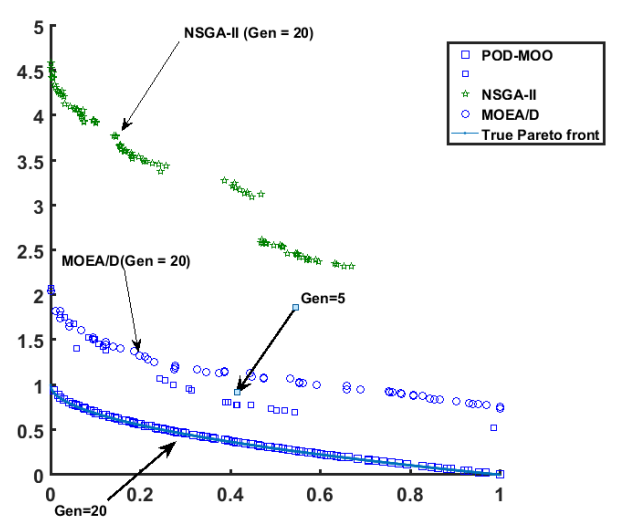

Figure 6 shows experimental results of the standard bi-objective ZDT series benchmarks: ZDT2, ZDT3, ZDT6, and ZDT4 ${ }^{1}$. Population size of the MOO is set to 200. Reference vectors of the decomposed bi-objective optimization (w in eq.17) are listed in the test of ZDT4. Although the benchmarks have different Pareto fronts, in each test, the MOO takes dozens of iterations to converge to the true Pareto front. In contrast, if the conventional genetic algorithms, e.g. NSGA-II are used, it may takes hundreds of iterations for the algorithms to search the true Pareto fronts.

\subsection{Surrogate Assisted Optimization}

To reduce the computational cost of the numerical model, the Kriging based surrogate can be used. The Kriging surrogate acts like an interpolator. First, a set of sample data and corresponding responses of the numerical model are put into the surrogate to train the surrogate. New data at the untested point can then be predicted using the Kriging surrogate.

The surrogate is constructed as follows. Consider a set of sample individuals $\mathbf{X}$ with the responses of the numerical model, $\mathbf{y}$, the Kriging prediction at point $\mathbf{x}$ can be constructed as a mean function plus a variance $[9,5]$

\footnotetext{
${ }^{1}$ Program code of POD-MOO and experimental simulations of the bi-objective and three objective benchmarks can be available at https://sites.google.com/site/adloptimization/moo-with-principlecomponent-analysis
} 

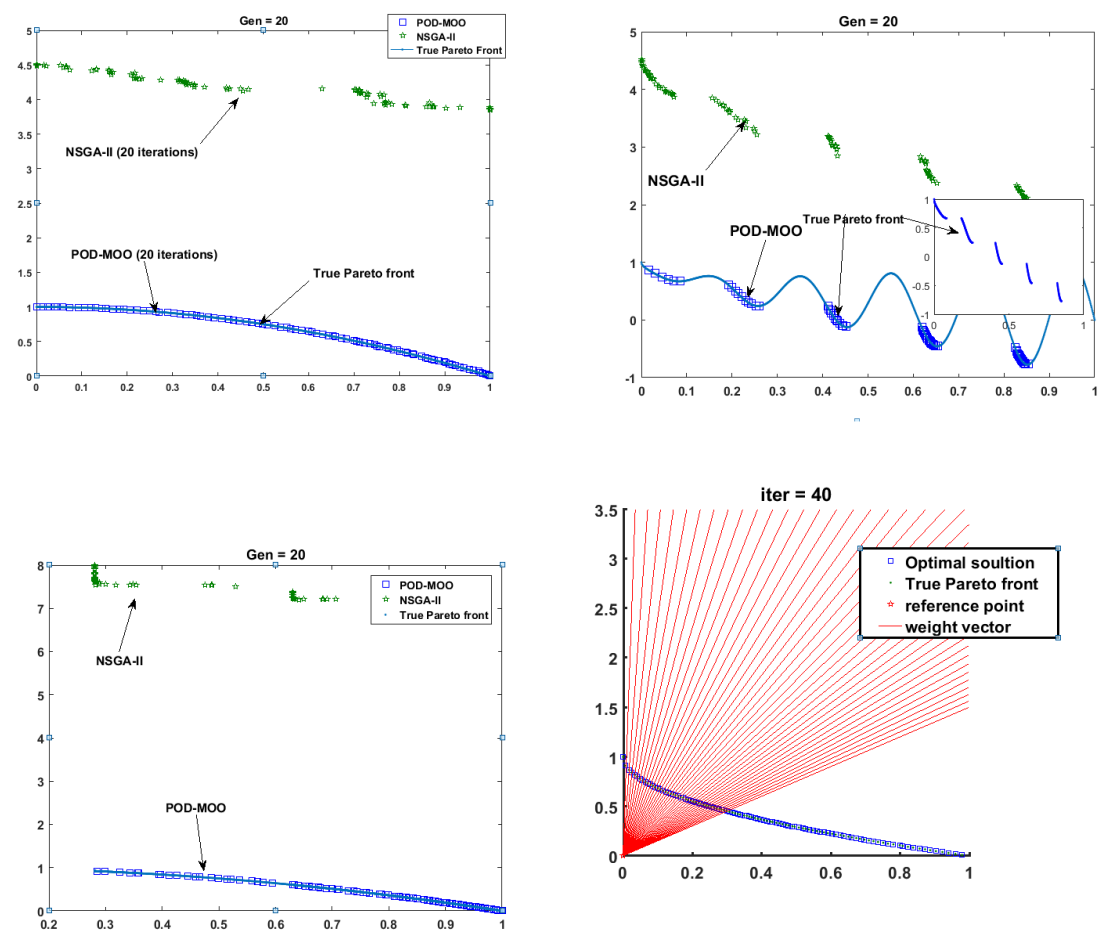

Fig. 6 Tests of POD-MOO on standard benchmarks of ZDT2(top-left), ZDT3 (top-right), ZDT6 (bottom-left) and ZDT4 (bottom-right). Population size: 200. Reference vectors of the decomposed bi-objective optimization ( $\mathbf{w}$ in eq.17) are listed in the test of ZDT4. Although the benchmarks have different Pareto fronts, in each test, the MOO takes dozens of iterations to converge to the true Pareto front.

$$
\hat{y}(\mathbf{x})=\hat{\mu}+\psi(\mathbf{x}, \mathbf{X}) \Psi^{-1}(\mathbf{X}, \mathbf{X})(\mathbf{y}-\mathbf{1} \hat{\mu})
$$

with the predictive mean and variance

$$
\begin{array}{r}
\hat{\mu}=\frac{\mathbf{1}^{T} \Psi^{-1}(\mathbf{X}, \mathbf{X}) \mathbf{y}}{\mathbf{1}^{T} \Psi^{-1}(\mathbf{X}, \mathbf{X}) \mathbf{1}} \\
\hat{\sigma}^{2}=\frac{(\mathbf{y}-\mathbf{1} \mu)^{T} \Psi^{-1}(\mathbf{y}-\mathbf{1} \mu)}{n}
\end{array}
$$

where 1 is the $n \times 1$ column vector of ones. $\psi(\mathbf{x}, \mathbf{X})$ and $\Psi\left(\mathbf{X}, \mathbf{X}^{\prime}\right)$ are the correlation vector and correlation matrix with

$$
\psi\left(\mathbf{x}, \mathbf{x}^{\prime}\right)=\exp \left(-\sum_{i=1}^{p_{i}} \theta_{i}\left|\mathbf{x}_{i}-\mathbf{x}_{i}^{\prime}\right|^{p_{i}}\right)
$$


where $\theta, p$ are the hyper parameters that can be determined using the maximum likelihood method $[9,5]$.

The mean squared error of the prediction at $\mathbf{x}$ is

$$
\hat{s}^{2}(\mathbf{x})=\sigma^{2}\left[1-\psi^{T} \Psi^{-1} \psi+\frac{1-\mathbf{1}^{T} \Psi^{-1} \psi}{\mathbf{1}^{\mathrm{T}} \Psi^{-1} \mathbf{1}}\right]
$$

To update the surrogate during the optimization, some more sample data can be used to train the surrogate further. In this work, an Expected Improvement (EI ) based infill strategy is used to update the surrogate.

Suppose $\mathscr{N}\left(\hat{y}(\mathbf{x}), \hat{s}^{2}(\mathbf{x})\right)$ is the Kriging prediction for the objective function $y(\mathbf{x})$, and the minimum of $y(\mathbf{x})$ over all evaluated points in population $\mathbf{X}$ is $y_{\min }$, then the expected improvement of an untested point $\mathbf{x}$ is [5]

$$
E I(\mathbf{x})=E\left[\max \left\{y_{\min }-y(\mathbf{x}), 0\right\}\right]
$$

and can be computed as

$$
E[I(\mathbf{x})]=\left[y_{\min }-\hat{y}(\mathbf{x})\right] \Phi\left(\frac{y_{\min }-\hat{y}(\mathbf{x})}{\hat{s}(\mathbf{x})}\right)+\hat{s}(\mathbf{x}) \phi\left(\frac{y_{\min }-\hat{y}(\mathbf{x})}{\hat{s}(\mathbf{x})}\right)
$$

New infill points can then be determined by exploring the design space using the infill criterion, and those points that have maximum EI values can be sampled as the infill points to improve the surrogate.

\subsection{Surrogate Assisted Evidence-Based Robust Design Optimization}

The design optimization consists of two loops. In the inner loop, the Kriging based surrogate of the expensive numerical lifetime predictor is constructed, while in the outer loop the new MOO algorithm is implemented. The surrogate reads the debris area-to-mass ratio (AMR), semi-axis, eccentricity, argument of perigee and mean anomaly and computes the debris lifetime till the decay altitude reaches $65 \mathrm{~km}$. Data of the numerical lifetime predictor are Latin Hypercube sampled and put into the surrogate to train the surrogate.

The evidence computation samples the uncertain space and decomposes the uncertain space into a series of subspaces. Uncertain parameters and the design variables are put into the surrogate to compute the lifetime. With the predicted lifetimes, evidence of the lifetimes can be computed using the sampling approach as eq.14 and eq. 15 show. The MOO of eq. 17 - eq.27 is then used to optimize the objective functions of the belief and lifetimes. Fig. 7 shows the flowchart of the proposed evidence-based robust design optimization. 


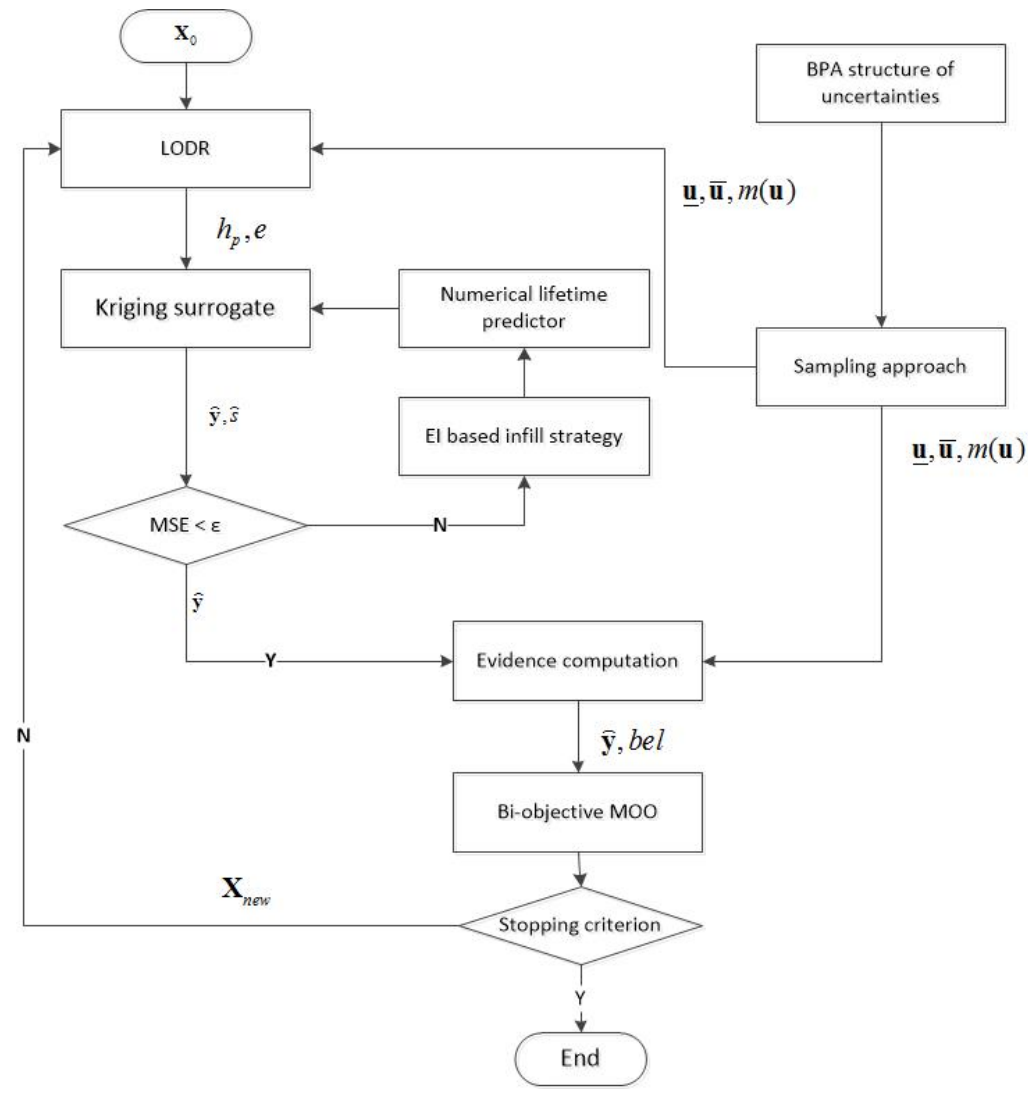

Fig. 7 Flowchart of the evidence-based robust design optimization. The design optimization consists of two loops. In the inner loop, the surrogate is constructed and updated with the new infill points, while in the outer loop, the bi-objective optimization is implemented to optimize debris lifetimes and their belief values.

\section{Numerical simulation}

Consider the robust design optimization problem of the pulsed LODR ${ }^{2}$. The target debris is set to Iridium-33, and the optimization objective is set to maximize under uncertainty the difference in the sum of the lifetimes of 10 debris objects. The orbital elements of the debris are from the CelesTrak database (TLEs of the debris are available at http://celestrak.com/NORAD/elements/iridium-33-debris.txt). Table 3 shows the parameters of the pulsed laser.

In the numerical propagator of the debris lifetime prediction, solar radiation pressure, atmospheric drag, non-spherical Earth and gravity from the third body are

${ }^{2}$ Part of program code of the robust POD-MOO and numerical simulation of LODR can be available at https://sites.google.com/site/adloptimization 
Table 3 Prameters of the pulsed LODR

\begin{tabular}{lll}
\hline Parameter & Value & Unit \\
\hline Number of impulse & 4750 & - \\
Laser pulse frequency & 100 & $\mathrm{~Hz}$ \\
Laser pulse interval & 100 & $\mathrm{~ns}$ between pulses \\
Laser power & 1000 & $\mathrm{~J} /$ pulse \\
\hline
\end{tabular}

taken into account. The Jacchia 1970 atmospheric density model is used, and the decay altitude is set to $65 \mathrm{~km}$. In the numerical lifetime predictor, a Runge-KuttaFehlberg 7th order integrator with 8th order error control is used. The drag coefficient is set to 2.2, and the coefficient of reflectivity is set to 0.28 [14]. Note that both values are closely related to the shape of the debris, and can vary with different debris. Regarding the LODR characteristic parameters, the coupling coefficient is $C_{m}=2.0 \cdot 10^{-5} \mathrm{Ns} / \mathrm{J}$ for the ideal case of aluminium, and the ablation rate is equal to $\mu=80 \cdot 10^{-9} \mathrm{~kg} / \mathrm{J}[20]$. Both the values could vary with the laser parameters such as intensity, wavelength, pulse length irradiated to the debris material, and surface conditions [20]. The average mass of a single Iridium-33 debris object is set equal to $m_{0}=1.327 \mathrm{~kg}$ [12]. A BPA structure for the parameters is presented (Table 3) to show the uncertainty effects. Three groups of estimated values and standard deviations are listed in the table, assuming that the uncertainties follow a Gaussian distribution.

Table 4 BPA structure of uncertainties. The uncertainties are of the LODR and debris parameters, and supposed to be estimated by three experts.

\begin{tabular}{|c|c|c|c|c|c|}
\hline Parameter & Description & $\mu$ & $\sigma_{l}$ & $\sigma_{u}$ & $\mathrm{BPA}$ \\
\hline & & $2.0 \cdot 10^{-5}$ & $0.8 \cdot 10^{-7}$ & $1.5 \cdot 10^{-7}$ & 0.60 \\
\hline \multirow[t]{3}{*}{$C_{m}(\mathrm{NS} / \mathrm{J})$} & Coupling coefficient & $2.8 \cdot 10^{-5}$ & $0.5 \cdot 10^{-7}$ & $1.0 \cdot 10^{-7}$ & 0.30 \\
\hline & & $2.0 \cdot 10^{-5}$ & $0.2 \cdot 10^{-7}$ & $1.2 \cdot 10^{-7}$ & 0.10 \\
\hline & & $80 \cdot 10^{-9}$ & $10 \cdot 10^{-10}$ & $20 \cdot 10^{-10}$ & 0.40 \\
\hline \multirow[t]{3}{*}{$u(\mathrm{~kg} / \mathrm{J})$} & Ablation rate & $78 \cdot 10^{-9}$ & $15 \cdot 10^{-10}$ & $22 \cdot 10^{-10}$ & 0.30 \\
\hline & & $72 \cdot 10^{-9}$ & $12 \cdot 10^{-10}$ & $17 \cdot 10^{-10}$ & 0.30 \\
\hline & & 1.30 & 0.05 & 0.10 & 0.30 \\
\hline \multirow[t]{3}{*}{$m_{0}(\mathrm{~kg})$} & Average mass [12] & 1.50 & 0.05 & 0.12 & 0.20 \\
\hline & & 1.42 & 0.05 & 0.08 & 0.50 \\
\hline & & 12.33 & 1.05 & 1.50 & 0.50 \\
\hline \multirow[t]{3}{*}{$r_{0}(\mathrm{~cm})$} & Debris average radius & 11.40 & 0.85 & 1.02 & 0.20 \\
\hline & & 13.52 & 0.75 & 0.98 & 0.30 \\
\hline & & 0.20 & 0.05 & 0.10 & 0.20 \\
\hline \multirow[t]{3}{*}{$C_{r}$} & coefficient of reflectivity & 0.23 & 0.07 & 0.12 & 0.30 \\
\hline & & 0.32 & 0.05 & 0.08 & 0.50 \\
\hline & & 2.20 & 0.05 & 0.10 & 0.30 \\
\hline \multirow[t]{2}{*}{$C_{d}$} & Drag coefficient & 2.23 & 0.05 & 0.12 & 0.20 \\
\hline & & 2.32 & 0.05 & 0.08 & 0.50 \\
\hline
\end{tabular}


Fig. 8 Solution A and B: Target debris, lifetimes, and belief values

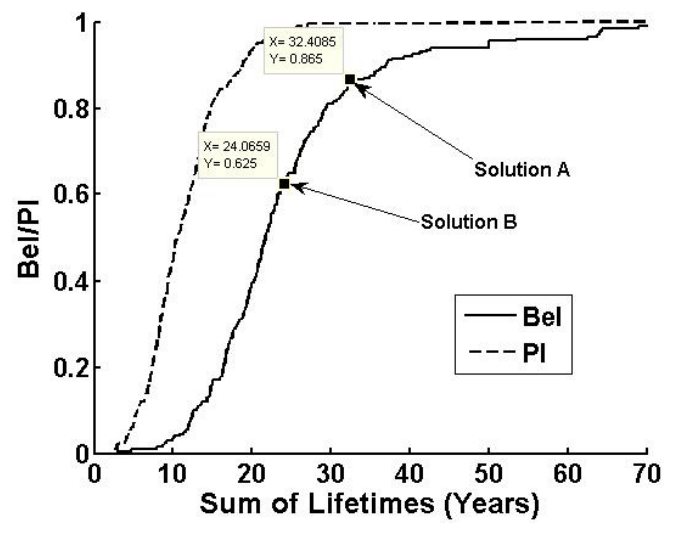

Most of the orbital eccentricities of the debris are less than 0.01, and the laser impulses are delivered within a short period of time (47 seconds in the simulation), therefore the accumulated velocity increment with a constant delivered angle can be used to compute the orbital elements after LODR. The station is set to Svalbard with latitude equal to 78.21670 degrees. To simplify the computation, the velocity is computed in advance using the parameters provided in Table 3 , and an equivalent constant $\vartheta, 120$ degrees is used (Figure 1 ).

All debris is associated to an ID number (No.1-466). The design vectors are then the ID numbers of the debris and the objective is set to maximize under uncertainty the difference in the sum of the lifetimes of 10 debris objects. Writing in mathematical form, the problem can be formulated as

$$
\min _{\mathbf{x} \in \mathbf{D}, \mathbf{u} \in \mathbf{U}} f(\mathbf{x}, \mathbf{u})=\sum_{i=1}^{10} L\left(\mathbf{x}_{i}, \mathbf{u}\right)
$$

where $\mathbf{x} \in \mathbb{N}$ are the indices of debris from 1 to $466, \mathbf{u} \in \mathbf{U}$ is the uncertain parameters, and $L\left(\mathbf{x}_{i}, \mathbf{u}\right)$ is the predicted lifetime of $\mathbf{x}_{i}$ debris.

The population size of robust POD-MOO is set to 200. Focal elements and corresponding masses are combined and approximated in advance to reduce the computational cost. The number of focal elements is set to be equal to the population size. A data set of numerical lifetime prediction with perigee altitude $h_{P} \in[250 \mathrm{~km}, 600 \mathrm{~km}]$ and eccentricity $e \in[0,0.3]$ is Latin-Hypercube sampled and put into Kriging to initialize the surrogate. The sample size is set to 240 . In each iteration, 5 solutions with maximum EI values are selected as the infill points, and put into the Kriging to update the surrogate.

Figure 8 shows the objective values of the optimal solutions after 50 iterations. Among the solutions, two solutions A and B with the belief of 0.865 and 0.625 respectively are selected. Table 5 shows the debris IDs of solution A and B, and their evidence levels. The belief of solution A is 0.865 for a sum of the post-LODR lifetimes of 32.41 years, while the belief of solution B is $0.625 / 24.07$ years. 
Table 5 Robust optimal solution A and B: Target debris, lifetimes before/after LODR, and evidence level of the optimal solutions.

\begin{tabular}{|c|c|c|c|}
\hline \multicolumn{2}{|c|}{ Solution Debris } & \multirow{2}{*}{$\begin{array}{l}\text { Sum (before/after, years) } \\
796.95 / 32.41\end{array}$} & \multirow{2}{*}{$\frac{\text { Belief/Plausibility }}{0.865 / 0.995}$} \\
\hline A & $283,115,9,31,3,75,425,192,112,52$ & & \\
\hline B & $45,133,109,308,15,275,297,144,82,40$ & $631.18 / 24.07$ & $0.625 / 0.975$ \\
\hline
\end{tabular}

To verify the robust solutions, a genetic based deterministic optimizer is used to optimize directly the objective function with $C_{m}=2.0 \cdot 10^{-5}, \mu=80 \cdot 10^{-9} \mathrm{~kg} / \mathrm{J}$, $m_{0}=1.327 \mathrm{~kg}$ respectively. As for coefficient of reflectivity, drag coefficient, and average radius of the debris, the value of $C_{r}=0.28, C_{d}=2.2, r_{0}=12.33 \mathrm{~cm}$ are used $[14,12]$.

Table 6 shows the deterministic optimal solution (Solution C) using the time consuming numerical predictor. The data is in good agreement with the proposed method and is highly consistent with the robust calculations when the uncertainty impacts are unconsidered. In Fig. 8, the minimum lifetimes of the curves are about 1.5 years with nearly zero belief (the belief is 0.04 ) in which the non-deterministic effects can be ignored, while the result of the deterministic approach is 1.558 years. The two sets of calculations are quite close. As can be seen, in addition to providing a deterministic calculation result, the proposed method can provide the confidence levels of the calculation results under non-deterministic conditions. All of these provide convenience for decision makers to optimize their designs.

Table 6 Solution C: Target debris and lifetimes before and after LODR (assuming all uncertainties are set to zero and using a deterministic optimizer)

\begin{tabular}{llll}
\hline Debris & Before (years) & After (years) & Sum (before/after, years) \\
\hline 142 & 8.32 & 0.07 & \\
194 & 10.97 & 0.16 & \\
168 & 29.01 & 0.44 & \\
207 & 7.60 & 0.11 & \\
133 & 9.82 & 0.18 & \\
335 & 8.17 & 0.11 & \\
142 & 8.32 & 0.07 & \\
281 & 8.96 & 0.03 & \\
196 & 22.63 & 0.32 & \\
159 & 3.67 & 0.04 & \\
\hline
\end{tabular}




\section{Conclusion}

In this work, an early stage robust analysis tool for the LODR design is presented. In the design optimization, both the epistemic uncertainty and aleatory uncertainty are considered. The evidence-based analysis tool is used to quantify the evidence level of the optimal solutions. Compared to the optimization method using conventional optimization algorithms, the method proposed in this work provides, not only the solutions that could be implemented in the LODR, but also the maximum belief values that the solutions can be achieved.

To resolve the problems due to the evidence computation and the expensive numerical model, a high efficiency MOO, using the Tchebysheff decomposition strategy and Gaussian predictor, is proposed. The MOO outperforms the conventional algorithms in a series of standard test functions. A surrogate is used to reduce the cost due to the expensive model. Evidence computations of the uncertainty impacts are integrated into the optimization via the sampling approach. At each iteration, the number of objective function evaluations is twice the population size. Data from the surrogate are used instead of the numerical expensive data, and an EI based infill strategy is used to update the surrogate.

In the simulation, the debris fragments are supposed to be spherical and face-on planes. As the lifetimes of the debris can vary greatly with the relevant parameters, a more detailed analysis of the objects properties, such as the realistic shapes and tumbling effects should be taken into account if an actual LODR is implemented. The robust optimization method presented in this chapter provides an early stage robust analysis tool for the LODR in order to determine the potential candidates. Impacts due to the attitude and shape of the debris will be taken into account in future works.

\section{References}

1. Agarwal, H., Renaud, J.E., Preston, E.L., Padmanabhan, D.: Uncertainty quantification using evidence theory in multidisciplinary design optimization. Reliability Engineering \& System Safety 85(1), 281-294 (2004)

2. Asafuddoula, M., Ray, T., Sarker, R.: A decomposition-based evolutionary algorithm for many objective optimization. IEEE Transactions on Evolutionary Computation 19(3), 445-460 (2015)

3. Auer, E., Luther, W., Rebner, G., Limbourg, P.: A verified matlab toolbox for the dempstershafer theory. In: Workshop on the Theory of Belief Functions (2010)

4. Balakrishnan, N.: Continuous multivariate distributions. Wiley Online Library (2006)

5. Couckuyt, I., Dhaene, T., Demeester, P.: oodace toolbox: a flexible object-oriented kriging implementation. Journal of Machine Learning Research 15, 3183-3186 (2014)

6. Croisard, N., Vasile, M., Kemble, S., Radice, G.: Preliminary space mission design under uncertainty. Acta Astronautica 66(5), 654-664 (2010)

7. Deb, K.: Multi-objective optimization. In: Search methodologies, pp. 403-449. Springer (2014)

8. Deb, K., Pratap, A., Agarwal, S., Meyarivan, T.: A fast and elitist multiobjective genetic algorithm: Nsga-ii. IEEE transactions on evolutionary computation 6(2), 182-197 (2002) 
9. Forrester, A., Keane, A., et al.: Engineering design via surrogate modelling: a practical guide. John Wiley \&amp; Sons (2008)

10. Hou, L., Pirzada, A., Cai, Y., Ma, H.: Robust design optimization using integrated evidence computationłwith application to orbital debris removal. In: Evolutionary Computation (CEC), 2015 IEEE Congress on, pp. 3263-3270. IEEE (2015)

11. Hou, L., Tan, W., Ma, H.: Multi-fidelity design optimization under epistemic uncertainty. In: Evolutionary Computation (CEC), 2016 IEEE Congress on, pp. 4452-4459. IEEE (2016)

12. Kelso, T., et al.: Analysis of the iridium 33-cosmos 2251 collision. Advances in the Astronautical Sciences 135(2), 1099-1112 (2009)

13. Liedahl, D., Rubenchik, A., Libby, S.B., Nikolaev, S., Phipps, C.R.: Pulsed laser interactions with space debris: target shape effects. Advances in Space Research 52(5), 895-915 (2013)

14. Mason, J., Stupl, J., Marshall, W., Levit, C.: Orbital debris-debris collision avoidance. Advances in Space Research 48(10), 1643-1655 (2011)

15. Moon, T.K., Stirling, W.C.: Mathematical methods and algorithms for signal processing, vol. 1. Prentice hall New York (2000)

16. Phipps, C., Albrecht, G., Friedman, H., Gavel, D., George, E., Murray, J., Ho, C., Priedhorsky, W., Michaelis, M., Reilly, J.: Orion: Clearing near-earth space debris using a 20-kw, 530-nm, earth-based, repetitively pulsed laser. Laser and Particle Beams 14(1), 1-44 (1996)

17. Phipps, C.R., Baker, K.L., Libby, S.B., Liedahl, D.A., Olivier, S.S., Pleasance, L.D., Rubenchik, A., Trebes, J.E., George, E.V., Marcovici, B., et al.: Removing orbital debris with lasers. Advances in Space Research 49(9), 1283-1300 (2012)

18. Rebner, G., Auer, E., Luther, W.: A verified realization of a dempster-shafer based fault tree analysis. Computing 94(2), 313-324 (2012)

19. Roy, A.E.: Orbital motion. CRC Press (2004)

20. Schall, W.O.: Laser radiation for cleaning space debris from lower earth orbits. Journal of spacecraft and rockets 39(1), 81-91 (2002)

21. Vasile, M.: Robust mission design through evidence theory and multiagent collaborative search. Annals of the New York Academy of Sciences 1065(1), 152-173 (2005)

22. Vasile, M.L.: A behavioral-based meta-heuristic for robust global trajectory optimization. In: Evolutionary Computation, 2007. CEC 2007. IEEE Congress on, pp. 2056-2063. IEEE (2007)

23. Volkwein, S.: Proper orthogonal decomposition: Theory and reduced-order modelling. Lecture Notes, University of Konstanz 4(4) (2013)

24. Xiao, D., Fang, F., Buchan, A.G., Pain, C.C., Navon, I.M., Du, J., Hu, G.: Non-linear model reduction for the navier-stokes equations using residual deim method. Journal of Computational Physics 263, 1-18 (2014)

25. Xie, D., Xu, M., Dowell, E.H.: Proper orthogonal decomposition reduced-order model for nonlinear aeroelastic oscillations. AIAA journal (2014)

26. Zhang, Q., Li, H.: Moea/d: A multiobjective evolutionary algorithm based on decomposition. IEEE Transactions on evolutionary computation 11(6), 712-731 (2007)

27. Zuiani, F., Vasile, M., Gibbings, A.: Evidence-based robust design of deflection actions for near earth objects. Celestial Mechanics and Dynamical Astronomy pp. 1-30 (2012) 